Europhys. Lett., 60 (6), pp. 889-895 (2002)

\title{
Coupling sensitivity of localization length in one-dimensional disordered systems
}

\author{
R. Zillmer, V. Ahlers and A. Pikovsky \\ Department of Physics, University of Potsdam \\ Postfach 601553, D-14415 Potsdam, Germany
}

(received 13 August 2002; accepted 7 October 2002)

PACS. 72.15.Rn - Localization effects (Anderson or weak localization).

\begin{abstract}
We investigate Anderson localization in a quasi-one-dimensional disordered system consisting of weakly coupled statistically independent channels. The dependence of the localization length on the coupling $\kappa$ is shown to be singular: proportional to $1 /|\ln \kappa|$. Numerical evidence is given for coupled random chains with different statistics of site potential. An analytical approach is developed based on the Fokker-Planck treatment of the coupled stationary Schrödinger equations with Gaussian delta-correlated potentials.
\end{abstract}

Anderson localization, i.e. an exponential in average decay of the amplitude of the wave function in space, is a fundamental phenomenon in disordered systems [1-4]. It results from a combination of tunneling and interference effects at the motion of a particle in a random potential. In terms of condensed-matter physics, the disorder relates to impurities, vacancies and dislocations in the crystal lattice. The exponential localization of the wave function plays an important role in the understanding of the transition between insulating and metallic states of matter [5]. Of particular theoretical interest are (quasi-)one-dimensional systems, where the transfer matrix method can be employed and many properties of localization can be obtained analytically. Experimental realization for one-dimensional localization provide carbon nanotubes, which have become objects of growing interest, both in experiment and theory, during the last decade (see [6] and references therein).

The main characteristic of the Anderson localization is the localization length $l$. In particular, it is directly related to the conductivity of a disordered layer via the Landauer formula. For one-dimensional systems the localization length can be related to the Lyapunov exponent (LE) of the corresponding random dynamical system. The simplest example (and the starting point for the theory below) is the stationary 1D Schrödinger equation for a single particle,

$$
-\psi^{\prime \prime}(x)+U(x) \psi(x)=e \psi(x),
$$

where the disorder is due to the random potential $U(x)$. Usually, Gaussian white noise is presumed:

$$
\left\langle U\left(x_{1}\right) U\left(x_{2}\right)\right\rangle=2 \sigma^{2} \delta\left(x_{1}-x_{2}\right) .
$$

Considering (1) as a linear dynamical system describing the spatial evolution of the vector $\left(\psi, \psi^{\prime}\right)^{t}$, one can in analogy with usual Hamiltonian dynamical systems define two LEs, $\gamma>0$ (C) EDP Sciences 
and $-\gamma$, which describe the mean exponential growth of the norm $\|\psi\|=\sqrt{\psi^{2}+\psi^{\prime} 2}$ in space [4]. The localization length is just the inverse LE $l=1 / \gamma$; in the case when there are many LEs (as in coupled lattices below), the localization length is related to the smallestin-absolute-value one. If the potential is a $\delta$-correlated Gaussian random field, an analytical expression for the LE can be found [2]. Below, also the fluctuations of the norm of the vector $\left(\psi, \psi^{\prime}\right)^{t}$ are important; one can characterize them with the diffusion constant $D$ (cf. [7]):

$$
\left\langle(\ln \|\psi(x)\|-x \gamma)^{2}\right\rangle \propto 2 D x \quad \text { for } \quad x \longrightarrow \infty .
$$

The diffusion constant is related to the variance of the random potential by $D \sim \sigma^{2 / 3}$ in the band tails, whereas in the band centre the single-parameter scaling holds: $D \sim \gamma[7]$.

In this letter we investigate the behavior of the localization length in weakly coupled onedimensional disordered systems. We consider the following setup: two different statistically equivalent but independent disordered channels (each being described by the Schrödinger equation like (1), or by its discrete analog) are weakly coupled so that there is a small probability $\kappa$ for a particle to hop to a neighboring channel. We show that the localization length singularly increases for small $\kappa / \sigma^{2}$ :

$$
l-l_{0} \sim l_{0}^{2} \frac{D}{|\ln \kappa|} .
$$

Here $l_{0}$ is the localization length of the uncoupled channel, and $D$ is the diffusion constant defined in (2). The limit we explore, $\kappa / \sigma^{2} \ll 1$, does not match the regime considered for the same setup (two coupled channels) by Dorokhov [8], who calculated $l$ in the opposite limit of large couplings. Our approach is to derive the corresponding relation for the deviation of the LE due to coupling:

$$
\Delta \gamma \sim \frac{D}{|\ln \kappa|}
$$

This relation is known for dissipative dynamical systems as coupling sensitivity of chaos [9-12]. As we show below, the extension to the Hamiltonian dynamics (1) is rather tricky. From (4) a similar singular behavior for the typical conductance $\tilde{G}=\exp [\langle\ln G\rangle]$ follows. Indeed, by invoking the Landauer formula the typical conductance of a sample with length $L$ is proportional to $\exp [-2 L \gamma]$, what leads with (4) to $\tilde{G} / \tilde{G}_{0} \sim 1+2 L D /|\ln \kappa|$, where $\tilde{G}_{0}$ is the conductance at zero coupling. Let us mention that the generalized Localization lengths, defined as (see [4]) $L_{q}=\lim _{x \rightarrow \infty}(q x)^{-1} \ln \left\langle\|\psi(x)\|^{-q}\right\rangle$, for integer $q$ behave regularly at small couplings: $\Delta L_{q} \sim \kappa$. Hence the same behavior for the average conductivity follows: $\Delta\langle G\rangle \sim \kappa$.

Qualitatively, the singular dependence of the localization length on coupling can be understood as follows (cf. [12]). Consider two weakly coupled channels. The wave functions in both channels $\psi_{1}, \psi_{2}$ spatially decrease in average exponentially, with the same average rate (LE). However, due to different fluctuations in statistically independent channels, it may happen that the wave function in one channel attains much larger values than in the other one, i.e. $\left\|\psi_{1}\right\| /\left\|\psi_{2}\right\|>\kappa^{-1}$. In this case it is favorable for the wave function to concentrate in channel 1; this is accomplished by hopping from 2 to 1 . Then the decrease continues until the ratio between the wave functions will be again of order of $\kappa^{-1}$ and a new hopping occurs, etc. Because the values $\ln \left\|\psi_{1,2}\right\|$ perform random biased walks in space, a characteristic length to reach a distance $|\ln \kappa|$ between them is $x_{h} \approx(\ln \kappa)^{2} / D$; this is thus a characteristic distance between inter-channel hopping. At each hopping the quantity $\ln \|\psi\|$ increases by $\Delta(\ln \|\psi\|) \approx|\ln \kappa|$. Thus the logarithmic decay rate of the wave function decreases by $\Delta(\ln \|\psi\|) / x_{h} \approx D /|\ln \kappa|$, which corresponds to the relation (3). 
Below we shall first present numerical evidence for the singular behavior of the localization length, and then shall develop an analytic approach.

Numerical evidence of coupling sensitivity of localization length. - We start with a discrete Anderson model based on the dimensionless tight-binding Hamiltonian [3]

$$
-\psi_{m+1}-\psi_{m-1}+\epsilon_{m} \psi_{m}=e \psi_{m} .
$$

This Anderson model describes a quantum particle on a chain of sites with random local site energy $\epsilon_{m}$. The same equation appears also in the Kronig-Penny model (with a slightly different interpretation of terms $\epsilon_{m}, e$, see [2]).

Next we consider a quasi-1D model consisting of two coupled chains. The amplitudes of the wave functions are now given by $\psi_{n, m}$, where $n=1,2$ labels the chains. We assume that the chains are coupled via the nearest-neighbor hopping amplitude $\kappa \ll 1$, i.e. the inter-chain hopping probability is much smaller than the intra-chain one. The stationary Schrödinger equation of this system has the form of two coupled Anderson models (5):

$$
\begin{aligned}
& -\psi_{1, m+1}-\psi_{1, m-1}+\epsilon_{1, m} \psi_{1, m}+\kappa \psi_{2, m}=e \psi_{1, m}, \\
& -\psi_{2, m+1}-\psi_{2, m-1}+\epsilon_{2, m} \psi_{2, m}+\kappa \psi_{1, m}=e \psi_{2, m} .
\end{aligned}
$$

The local site energies $\epsilon_{1, m}, \epsilon_{2, m}$ are assumed to be independent equally distributed in both chains. Because the inter-chain coupling is close to zero, the model is not well described by the DMPK equation [13], which presumes isotropic scattering by neglecting the length scale of transverse diffusion. The spatial evolution of the state $\boldsymbol{\Psi}_{m} \equiv\left(\psi_{1, m}, \psi_{2, m}, \psi_{1, m-1}, \psi_{2, m-1}\right)^{t}$ is determined by a symplectic transfer matrix, $\boldsymbol{\Psi}_{m+1}=\boldsymbol{T}_{m} \boldsymbol{\Psi}_{m}$, and is thus area-preserving with two positive LEs $\gamma_{1}, \gamma_{2} \leq \gamma_{1}$, the negative exponents being related to the positive ones by $\gamma_{4,3}=-\gamma_{1,2}$. At vanishing coupling $\kappa=0$ the exponents $\gamma_{1}$ and $\gamma_{2}$ coincide; we denote the exponent of the uncoupled system as $\Lambda$.

The localization length of the whole lattice is given by the smallest-in-absolute-value LE: $l=1 / \gamma_{2}$. Our main interest is in the deviations of this length from that of uncoupled system (5) $1 / \Lambda$. We calculated the LEs by iterating the vector $\boldsymbol{\Psi}_{m}$ with the transfer matrix and reorthonormalizing it using a modified Gram-Schmidt algorithm. Additionally, for each setup of statistics we calculated the diffusion constant $D(2)$ by iterating the uncoupled system (5).

In fig. 1(a) we present the results for different distributions of site potential $\epsilon_{n, m}$ and different energies $e$. The figure clearly demonstrates singular splitting of the first and second LEs. Figure 1(b) shows the same data in the scaled coordinates: $\left(\gamma_{1,2}(\kappa)-\Lambda\right) / D$ vs. $1 /|\ln \kappa|$. Here the curves for the different distributions and the same $e$ collapse on straight lines, as expected according to eq. (4). The linear fit, also shown in the figure, reveals that the splitting is symmetric, i.e. $\gamma_{2}(\kappa)-\Lambda=-\left(\gamma_{1}(\kappa)-\Lambda\right)$. This symmetry, important for the analytical approach below, can be explained as follows. The sum of positive LEs is related to the density of states via the generalization of the Thouless formula [4]. Because the eigenstates non-singularly depend on the coupling, the sum of the positive LEs also depends on $\kappa$ in a nonsingular way. Therefore, the singular deviations of the first and the second LEs are symmetric.

The model (6) can be straightforwardly generalized to the case of $N$ coupled chains; we still assume that hopping amplitudes $\kappa$ between the chains are much less than those inside the chains; furthermore, periodic boundary conditions in the transverse direction are used: $\psi_{N+1, m}=\psi_{1, m}$. Now one has to deal with $N$ positive LEs which coincide for $\kappa=0$. (For the relation of these exponents to the electrical conductance properties see [14,15].) The rescaled plot of fig. 2(a) shows a different slope for the splitting of $\gamma_{1,4}$ and $\gamma_{2,3}$, respectively. Again the splitting is symmetric. The localization length determined by $\gamma_{4}$ thus increases according to eq. (3). 

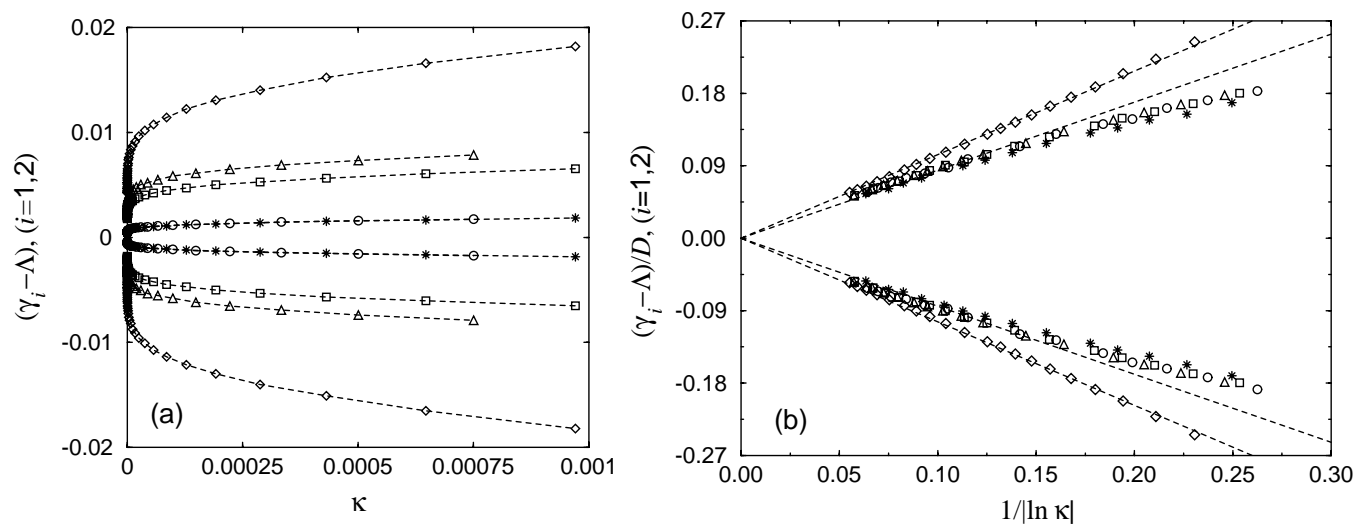

Fig. 1 - (a) The upper graphs show the splitting $\gamma_{1}(\kappa)-\Lambda$ vs. $\kappa$ at $e=-0.09$ for a two-point (circles), a Gaussian (stars) and a box distribution of $\epsilon_{k, m}$; the latter with widths $w=2.6$ (squares) and $w=3.0$ (triangles). At $e=2.5$ the splitting is shown for the box distribution with $w=1.6$ (diamonds). The lower graphs show $\gamma_{2}(\kappa)-\Lambda$ for the same values of $w$ and $e$. The dashed lines are to improve readability. (b) The same as (a) but in rescaled coordinates: $\left(\gamma_{1}(\kappa)-\Lambda\right) / D$ (upper graphs) and $\left(\gamma_{2}(\kappa)-\Lambda\right) / D$ (lower graphs) vs. $1 /|\ln \kappa|$. The dashed lines are linear fits with slopes \pm 0.85 for $e=-0.09$ and \pm 1.04 for $e=2.5$, respectively. The valid range of scaling (3) depends on $e$ : in the band centre it is $\kappa<10^{-5}$, while at the band edge it expands up to $\kappa \approx 10^{-3}$.

Coupling sensitivity also appears in the case of random coupling, which underlines the robustness of the effect. We investigated the following generalization of model (6):

$$
\begin{aligned}
& -\psi_{1, m+1}-\psi_{1, m-1}+\epsilon_{1, m} \psi_{1, m}+\kappa \xi_{1, m} \psi_{2, m}=e \psi_{1, m}, \\
& -\psi_{2, m+1}-\psi_{2, m-1}+\epsilon_{2, m} \psi_{2, m}+\kappa \xi_{2, m} \psi_{1, m}=e \psi_{2, m} .
\end{aligned}
$$

The random numbers $\xi_{n, m}$ are again assumed to be independent equally distributed in both chains. The rescaled plot of the positive LEs is shown in fig. 2(b) for two values of the energy. The linear fits confirm the singular behavior according to eq. (4).
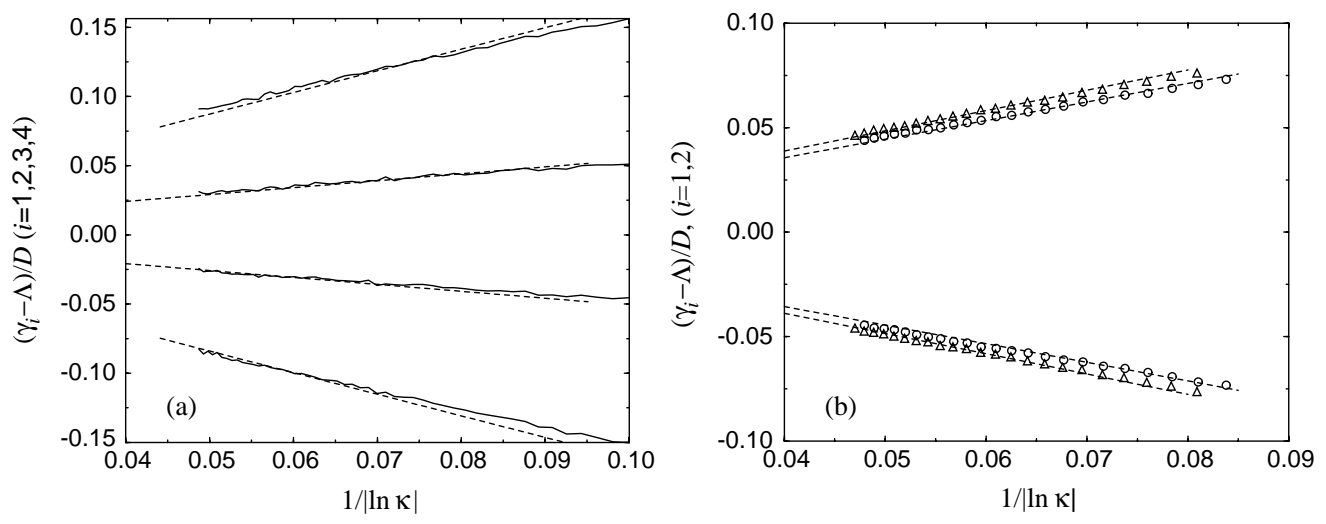

Fig. 2 - Splitting of the LEs in rescaled coordinates. (a) Four coupled channels (the parameters were $e=-0.08, w=1.6$; dashed lines have slope \pm 1.56 and \pm 0.44 , respectively). (b) Two channels with random coupling for $e=0$ (circles) and $e=-1.8$ (triangles). The dashed lines are linear fits with slopes \pm 0.89 and \pm 0.97 , respectively. The noise was chosen box-distributed with $w=\sqrt{3} / 2$. 


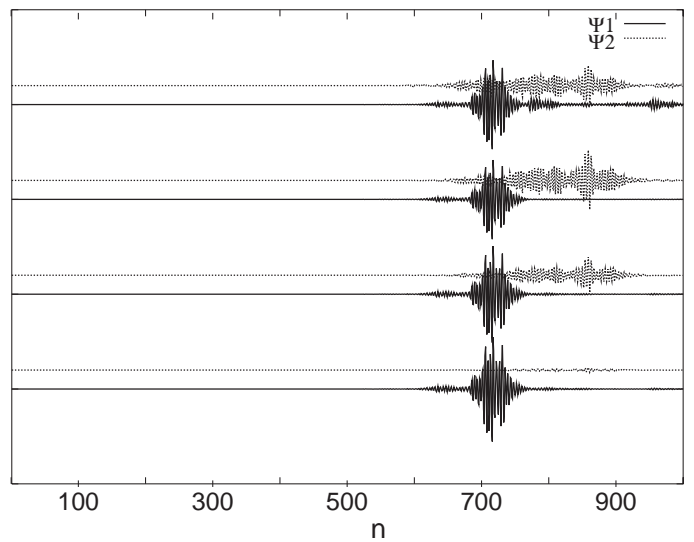

Fig. 3 - The amplitudes $\psi_{1, n}, \psi_{2, n}$ vs. $n$ for different values of $\kappa$. Chain 2 is shifted with respect to chain 1 , and this pair is shifted by a larger amount for the values (starting from the bottom) $\kappa=10^{-5}, 10^{-4}, 10^{-3}, 10^{-2}$, respectively.

Finally, we demonstrate how the localized states of weakly coupled chains look like. By numerically diagonalizing the tight-binding Hamiltonian, the amplitudes of the localized states have been obtained for different values of coupling. The number of lattice sites has been chosen as $N=2000$, i.e. 1000 per chain. In fig. 3 these amplitudes are plotted for the energy value $e=0.0944$ at $\kappa=0$ and the width of the box distribution $w=0.8$. It should be stressed that the plotted amplitudes result from one realization of the random potential on a quite short lattice length $N$, so that there is a strong influence of finite-size effects. At $\kappa=0$ there exists a localized state only on one chain because we are dealing with a one-particle Hamiltonian. With small coupling the hopping to the other chain occurs, as described qualitatively above. For larger coupling $\left(\kappa=10^{-2}\right)$ one can see also the back-hopping. As a result, small coupling leads to a considerable spread of the localized state.

Theory. - In the absence of random potential and coupling, the energy in (5) is given by $e=-2 \cos k$, with the wave vector $k$. For $e \rightarrow-2$ the wavelength diverges and the continuous model (1) may be employed. Below we develop an analytical approach for large negative energies $e<-2$, using the continuum version of (6):

$$
\begin{aligned}
& -\psi_{1}^{\prime \prime}+U_{1}(x) \psi_{1}+\kappa \psi_{2}=(e+2) \psi_{1}, \\
& -\psi_{2}^{\prime \prime}+U_{2}(x) \psi_{2}+\kappa \psi_{1}=(e+2) \psi_{2} .
\end{aligned}
$$

For convenience, we have set the lattice constant equal to unity. We consider the system (7) in the limit of small coupling $\kappa \ll 1$. The random potential is assumed to be a $\delta$-correlated Gaussian random variable: $\left\langle U_{i}\left(x_{1}\right) U_{j}\left(x_{2}\right)\right\rangle=2 \sigma^{2} \delta_{i j} \delta\left(x_{1}-x_{2}\right)$.

Without coupling the variables $v_{n}(x)=\psi_{n}^{\prime} / \psi_{n}$ perform a random walk in the potential $\Phi(v)=v^{3} / 3+(e+2) v$,

$$
v_{n}^{\prime}=-v_{n}^{2}-(e+2)+U_{n}(x) .
$$

A change of sign of $\psi_{n}$ corresponds to the limit $v_{n} \rightarrow-\infty$ followed by a reinjection at $v_{n}=$ $+\infty$; i.e. there is a probability flow proportional to the density of nodes of the eigenfunction. For $e<-2$ the potential $\Phi$ has a minimum at $v=\sqrt{|e+2|}$ with the Kramers escape time

$$
T_{\mathrm{K}}=|e+2|^{-1 / 2} \exp \left[4|e+2|^{3 / 2} / 3 \sigma^{2}\right] .
$$




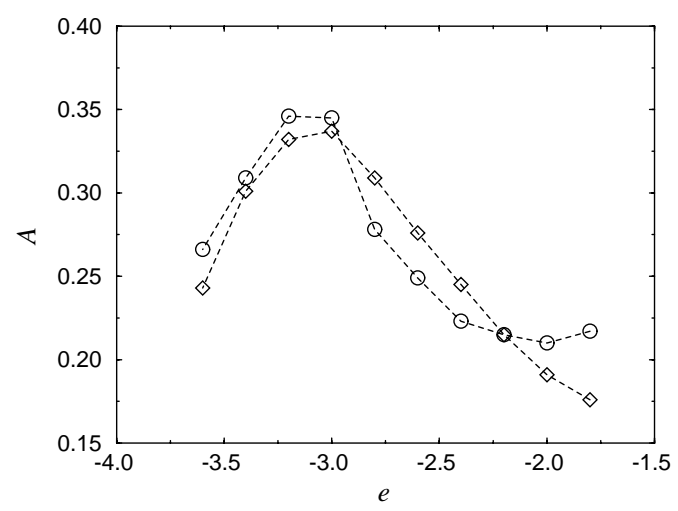

Fig. 4 - The strength $A$ vs. $e$ for box-distributed potential with width $w=\sqrt{3} / 2$. Shown are the numerical results (diamonds) and the analytical approach (circles).

For $T_{\mathrm{K}} \gg 1$, we may assume that the variable $v_{n}$ is trapped sufficiently long around the minimum and hence may presume $\psi_{n}>0$. We now introduce the variables $w_{1}=v_{1}-v_{2}$, $w_{2}=v_{1}+v_{2}$ and $z=\ln \left(\psi_{1} / \psi_{2}\right)$, whereupon the equations of motion can be rewritten as

$$
\begin{aligned}
w_{1}^{\prime} & =U_{1}-U_{2}-w_{1} w_{2}-2 \kappa \sinh z \\
w_{2}^{\prime} & =U_{1}+U_{2}-\frac{w_{1}^{2}+w_{2}^{2}}{2}-2(e+2)+2 \kappa \cosh z, \\
z^{\prime} & =w_{1} .
\end{aligned}
$$

The maximal Lyapunov exponent in terms of the new variables reads $\gamma_{1}=\left\langle(\ln \|\psi\|)^{\prime}\right\rangle=$ $\left\langle w_{2}\right\rangle / 2$. Note that, from the symmetry discussed above, $\gamma_{2} \approx 2 \Lambda-\gamma_{1}$, thus in this way we determine the scaling relevant for the localization length $l$.

Differentiating the equation of motion for $z$ yields

$$
z^{\prime \prime}=U_{1}-U_{2}-w_{2} z^{\prime}-2 \kappa \sinh z
$$

We now make a rather crude assumption that the variables $w_{1}$ and $w_{2}$ are weakly correlated. Hence a mean-field approach may be employed by replacing $w_{2}$ in (9) by its mean value $2 \gamma_{1}$. Then $z$ performs a Brownian motion in the potential $2 \kappa \cosh z$ and the stationary solution is given by the Boltzmann distribution

$$
\varrho(z) \propto \exp \left[-\frac{\gamma_{1}}{\sigma^{2}} 2 \kappa \cosh z\right] .
$$

Averaging the equations of motion $(8 \mathrm{a}),(8 \mathrm{~b})$ with $\varrho(z)$ leads to

$$
\begin{aligned}
& w_{1}^{\prime}=U_{1}-U_{2}-w_{1} w_{2}, \\
& w_{2}^{\prime}=U_{1}+U_{2}-\frac{w_{1}^{2}+w_{2}^{2}}{2}-2(e+2)+2 \frac{\sigma^{2} / 2 \gamma_{1}}{\left|\ln \left(2 \gamma_{1} \kappa / \sigma^{2}\right)\right|},
\end{aligned}
$$

for small $\kappa$. Hence the coupling corresponds to a small shift of the effective energy, whereupon the corresponding correction to the Lyapunov exponent of model (6) is given by

$$
\gamma_{1}(\kappa)=\Lambda+\frac{\mathrm{d} \Lambda}{\mathrm{d}|e|} \frac{\sigma^{2} / 2 \Lambda}{\left|\ln \left(2 \Lambda \kappa / \sigma^{2}\right)\right|}
$$


for $|e|>2$, where we used the fact that the largest LE is an increasing function of $|e|$. This result corresponds to the logarithmic singularity of the Lyapunov exponent (4).

To check the validity of formula (11) we compare in fig. 4 the prefactor $A$ in the relation $\gamma_{1}(\kappa)-\Lambda=A /|\ln \kappa|$ obtained from numerical simulations with the theoretical one. The analytical results reasonably agree with the numerical calculations, while the agreement is lost with the energy tending to the band centre. As already indicated by fig. 1(b), the strength increases with $|e|$ ascending from the band centre.

A similar approach can be accomplished for the opposite regime of large positive energies in (7), i.e. $e \gg \sigma^{2}$, which utilizes an averaging of terms oscillating with the large frequency $\sqrt{e}$. However, only the existence of the logarithmic singularity without explicitly determining the factor of the strength could be confirmed in this case.

Conclusion. - In this work we demonstrated that small coupling of one-dimensional disordered chains yields singular increase of the typical localization length. Qualitatively, the effect results from a consideration of coupled random walks, where the "walkers" are the logarithms of the norms of the wave function in the chains. In terms of the motion of a particle, the coupling gives rise to the possibility to tunnel between the disordered subsystems when one "walker" goes far ahead, i.e. when the conditions for the particle propagation in one chain are more favorable than in the other one. This leads already for very small couplings to a considerable spread of the wave function that appears as the singular increase of the localization length.

We have performed numerical simulations of the tight-binding model which has the form of coupled Anderson models and have demonstrated the singularity for two and four coupled chains. The results for the behavior of the LEs at small couplings agree with the analytical findings for the continuum disordered model, whereupon the effect seems to be a general phenomenon in systems with linear localization. It can be considered as a Hamiltonian analog of the coupling sensitivity of chaos in dissipative dynamical systems.

$$
* * *
$$

The work was supported by DFG (Sonderforschungsbereich 555 "Komplexe nichtlineare Prozesse").

\section{REFERENCES}

[1] Anderson P. W., Phys. Rev., 109 (1958) 1492.

[2] Lifshitz I. M., Gredeskul S. A. and Pastur L. A., Introduction to the Theory of Disordered Systems (Wiley, New York) 1988.

[3] Kramer B. and MacKinnon A., Rep. Prog. Phys., 56 (1993) 1469.

[4] Crisanti A., Paladin G. and Vulpiani A., Products of Random Matrices in Statistical Physics (Springer, Berlin) 1993.

[5] Schreiber M. and Grussbach H., Phys. Rev. Lett., 76 (1996) 1687.

[6] Kostyrko T., Bartkowiak M. and Mahan G. D., Phys. Rev. B, 60 (1999) 735.

[7] Deych L. I., Lisyansky A. A. and Altshuler B. L., Phys. Rev. Lett., 84 (2000) 2678.

[8] Dorokhov O. N., JETP Lett., 36 (1982) 318.

[9] Daido H., Prog. Theor. Phys., 72 (1984) 853.

[10] Livi R., Politi A. and Ruffo S., J. Phys. A, 25 (1992) 4813.

[11] Cecconi F. and Politi A., J. Phys. A, 32 (1999) 7603.

[12] Zillmer R., Ahlers V. and Pikovsky A., Phys. Rev. E, 61 (2000) 332.

[13] Beenakker C. W. J., Rev. Mod. Phys., 69 (1997).

[14] Pichard J.-L., PhD Thesis, Université de Paris Orsay, Orsay (1984).

[15] Kottos T., Izrailev F. M. and Politi A., Physica D, 131 (1999) 155. 